\title{
PARALIND-based blind joint angle and delay estimation for multipath signals with uniform linear array
}

\author{
Xu Liu ${ }^{1 *}$, Liang Guang ${ }^{2}$, Longxiang Yang ${ }^{1}$ and Hongbo Zhu ${ }^{1}$
}

\begin{abstract}
A novel joint angle and delay estimation (JADE) algorithm for multipath signals, based on the PARAllel profiles with LINear Dependencies (PARALIND) model, is proposed. Capitalizing on the structure property of Vandermonde matrices, PARALIND model is proved to be unique. Angle and delay of multiple rays of sources can be estimated by PARALIND decomposition and an ESPRIT-like shift-invariance technique. Simulation results show that the proposed algorithm outperforms the traditional JADE algorithm. It can automatically distinguish the estimated parameters between sources, and still be available when the number of rays is larger than the number of receiving antennae.
\end{abstract}

Keywords: Parameter estimation, Tensor decomposition, Multi-way analysis, PARALIND model

\section{Introduction}

With mobile communication stepping into the 3rd generation (3 G), smart antenna technique, which can combat multipath fading and improve system performance effectively, has already been applied to WCDMA and TDSCDMA communication systems [1,2]. In multipath scenario, wireless channel is characterized not only by their time delays of the different propagation paths, but also by their direction of arrivals (DOAs). The estimation of DOAs and time delays of the multipath rays is one major problem in smart antenna system to effectively locate and track various types of signals to minimize interference and maximize intended signal reception [3,4]. The focus of this article is on the joint estimation of angles and relative delays of multipath propagation signals emanating from sources and received by an antenna array. Various maximum likelihood (ML) approaches to joint angle and delay estimation (JADE) problems, such as the Expectation Maximization algorithm [5] and the Space-Alternating Generalized Expectation maximization [6] show superior performance in low signal-to-noise ratio (SNR) scenario when the number of samples is small or the sources are

\footnotetext{
* Correspondence: liuxu@njupt.edu.cn

'Jiangsu Key Laboratory of Wireless Communication, College of Telecommunications and Information Engineering, Nanjing University of Posts \& Telecommunications, Nanjing, China

Full list of author information is available at the end of the article
}

correlated. However, these approaches often require computationally ML searches, which is unattractive in online process. A number of subspace-based joint parameters estimation algorithms, which exploit the spatial or/and temporal structure assumptions and eigenvalue decomposition method, have also been derived. This type of JADE algorithms includes Multi-Dimensional Estimation of Signal Parameters via Rotational Invariance Technique (MD-ESPRIT) [7], Shift-Invariance Joint Angle and Delay Estimation (SIJADE) [8], and JADE-ESPRIT [9]. Most of JADE algorithms focus on single source in multipath environment and need perfect channel estimation. The authors of [10] expanded the idea of JADE-ESPRIT and proposed a blind JADE algorithm. Only a few of them allow the estimation of more paths than the number of receiving antennae available. In multiuser scenario, some traditional algorithms cannot distinguish the estimated parameters between sources [10].

Parallel factor (PARAFAC) is a subset of multi-way analysis and has initially been introduced as a data analysis tool in psychometrics and chemometrics. The authors of [11] first applied PARAFAC analysis into signal processing area. Nowadays, PARAFAC has widely been used in blind multiuser detection for CDMA system [12], array signal processing [13], OFDM system [14], and blind channel estimation [15]. Applications of PARAFAC analysis in wireless communication often 
assume single path propagation. ${ }^{\text {a }}$. In multipath propagation scenario, linear dependency factors usually occur in one or/and two mode matrices of the data model, where PARAFAC analysis will fail to provide meaningful results. PARAllel profiles with LINear Dependencies (PARALIND) is a generalization of PARAFAC and was developed to extend its usage to problems with linearly dependent factors [16]. In signal processing and communication applications of multi-way analysis, data with PARALIND structure arise in many problems due to what is known as spatial multipath, and where identical signals appear with different angles and delays. In these cases, by capitalizing on additional problem-specific structure, the associated PARALIND model can be proved to be unique. The authors of [17] applied PARALIND analysis in blind multiuser detection in WCDMA systems with large delay spread. Recently, PARALIND has been used in blind multiuser detection for smart antennae CDMA systems [18].

This article applies PARALIND analysis to the blind JADE problem. The received signals of the uniform linear array, transmitted through incoherent multipath rays of sources with distinct angles and delays, are constructed into PARALIND model. A new blind PARALIND-based JADE algorithm is proposed. This algorithm can be separated into two stages. The first stage applies PARALIND decomposition to the receiving data, thereby reducing the multiusermultipath parameters estimation problem to a simple singleuser-multipath parameters estimation problem. Then an ESPRIT-like shift-invariance technique is used to estimate the angles and delays of all paths corresponding to each user. The main advantages of our method are

(i) High parameter estimation performance. Simulations show that the PARALIND-based joint angle and delay estimator have better performance than traditional JADE algorithm.

(ii) Automatically distinguishing the estimated parameters between sources. The proposed estimator can automatically distinguish the estimated parameters of each source in multiuser scenario, which is not achieved, to the best of authors' knowledge, by traditional algorithms.

(iii) Being still valid when the number of rays is larger than the number of receiving antennae. The identifiability results of parameters, discussed in Section 3, demonstrate that the proposed algorithm allows the number of multipath to be larger than the number of receiving antennae.

The rest of this article is outlined as follows: Section 2 lays out the data model. Section 3 discusses the uniqueness results of PARALIND, and the identifiability results of parameters estimation are also provided. Section 4 proposes a new PARALIND-based blind JADE algorithm. Section 5 gives simulation results for performance evaluation. In the last section, we summarize conclusions.

Some notation conventions will be used in this article. $\operatorname{diag}([a, b \ldots])$ denotes the diagonal matrix with diagonal scalar entries $a, b, \ldots$ while block $\operatorname{diag}([\mathbf{A}, \mathbf{B} \ldots])$ denotes the block diagonal matrix with diagonal matrix entries $\mathbf{A}$, B. . ; $(\cdot)^{T}$ and $(\cdot)^{\dagger}$ stand for transpose and pseudoinverse, respectively; $\|\cdot\|_{F}^{2}$ stands for Frobenius norm; vec $(\cdot)$ stacks the columns of its matrix argument in a vector; unvec $(\cdot)$ is the inverse operation of $\operatorname{vec}(\cdot), \operatorname{unvec}(\mathbf{c}, I, J)=$ $[\mathbf{c}(1: J), \mathbf{c}(J+1: 2 J), \ldots, \mathbf{c}((I-1) J: I J)] ; \otimes$ is Kronecker product; $\odot$ denotes the Khatri-Rao product, which is a column-wise Kronecker product. Define $\mathbf{A}=\left[\mathbf{a}_{1}, \ldots, \mathbf{a}_{\boldsymbol{R}}\right] \in$ $\mathbb{C}^{I \times R} \mathbf{B}=\left[\mathbf{b}_{1}, \ldots, \mathbf{b}_{\boldsymbol{R}}\right] \in \mathbb{C}^{J \times R}$. The Khatri-Rao product of $\mathbf{A}$ and $\mathbf{B}$ is

$$
\mathbf{A} \odot \mathbf{B}=\left[\mathbf{a}_{1} \otimes \mathbf{b}_{1}, \ldots, \mathbf{a}_{R} \otimes \mathbf{b}_{R}\right]
$$

\section{Data model}

Consider a schematic communication scenario with multipath channel, depicted in Figure 1.

$M$ sources are transmitting to an array with $K$ antennae through multipath scattering propagation channel. Signals of source $m$ follow $r_{m}$ distinct paths on its way to the receiver, referred to as multipath rays with distinct direction of arrival, transmitting delay and attenuation. The multipath fading channel between source $m$ and receiving antennae array can be characterized as a $K \times 1$ vector $\mathbf{h}_{m}(t)$ :

$$
\mathbf{h}_{m}(t)=\sum_{j=1}^{r_{m}} \mathbf{a}\left(\theta_{m, j}\right) \beta_{m, j} g\left(t-\tau_{m, j}\right)
$$

In this model, the $j$ th path of source $m$ is parameterized by a triple $\left(\theta_{m, j}, \beta_{m, j}, \tau_{m, j}\right)$, where $\theta_{m, j}$ is the direction of arrival, $\beta_{m, j}$ is the complex path attenuation, $\tau_{m, j}$ is the time delay, $\mathbf{a}(\theta)$ is the array response vector of $K \times 1$, corresponding to signals from direction $\theta$. We assume that the uniform linear array is used in the receiving end and the distance $d$ between adjacent elements is equal to half the wavelength of signals. $\mathbf{a}(\theta)$ has the following form:

$$
\mathbf{a}(\theta)=\left[\begin{array}{c}
a_{1}(\theta) \\
a_{2}(\theta) \\
\vdots \\
a_{K}(\theta)
\end{array}\right]=\left[\begin{array}{c}
e^{-j \frac{2 \pi \hat{d}}{\lambda} \sin (\theta)} \\
\vdots \\
e^{-j \frac{2 \pi(K-1) d}{\lambda}} \sin (\theta)
\end{array}\right]
$$

$g(t)$ is the impulse response which collects all temporal aspects, such as pulse shaping, transmit filter, and receive filter. In incoherent multipath with small delay spread, we 


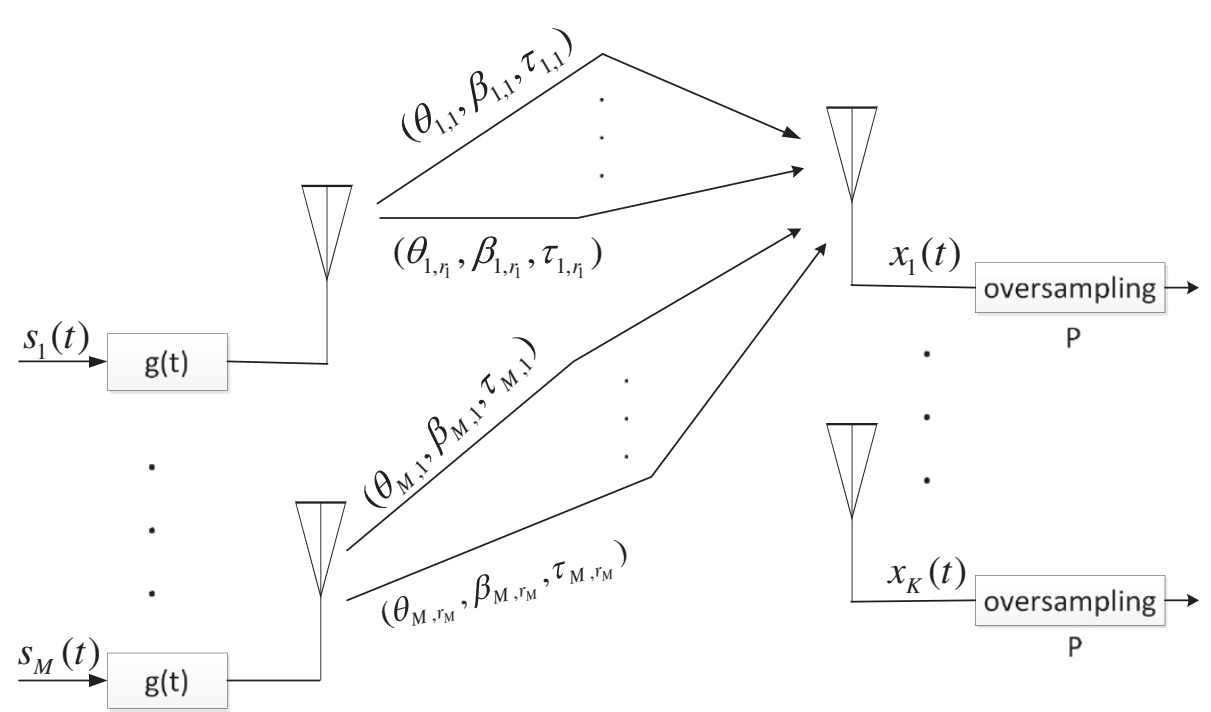

Figure 1 Multipath propagation channel model.

assume that $g(t)$ is zero outside an interval $\left[0, L_{g} \mathrm{~T}_{s}\right]$ with $L_{g}<1$, where $\boldsymbol{T}_{s}$ is the symbol period. The delay spread $\tau$ is relatively small and $L_{g} \boldsymbol{T}_{s}+\tau<\boldsymbol{T}_{s}$. This means that the sample of the received signal is a combination of $M$ and no more than $M$ sources symbols [10]. At this point, the received baseband signals at the output of the antenna array can be expressed as follows

$$
\begin{aligned}
\mathbf{x}(t) & =\sum_{m=1}^{M} \mathbf{h}_{m}(t) * \mathbf{s}_{m}(t) \\
& =\sum_{m=1}^{M} \sum_{i=1}^{r_{m}} \mathbf{a}\left(\theta_{m, i}\right) \beta_{m, i} g\left(t-\tau_{m, i}\right) s_{m}(t)
\end{aligned}
$$

where $s_{m}(t)$ is transmitted signal of $m$ th source at time $t$. ' $*$ ' denotes convolution operator. We oversamplex $(t)$ at a rate of $P$ times of symbol rate. Collect samples during $N$ symbol periods and construct a $K P \times N$ space-time data matrix

$$
\mathbf{X}=\left[\begin{array}{lll}
\mathbf{x}(0) \mathbf{x}(1) & \ldots \mathbf{x}(N-1) \\
\mathbf{x}\left(\frac{1}{P}\right) & \mathbf{x}\left(1+\frac{1}{P}\right) \ldots \mathbf{x}\left(N-1+\frac{1}{P}\right) \\
\vdots & \vdots & \vdots \\
\mathbf{x}\left(\frac{P-1}{P}\right) & \mathbf{x}\left(1+\frac{P-1}{P}\right) & \ldots \mathbf{x}\left(N-1+\frac{P-1}{P}\right)
\end{array}\right]
$$

Define $r=\sum_{m=1}^{M} r_{m}$ is the total number of paths of all sources. Let us conveniently index the rays from 1 to $r$, starting with all rays associated with the first source and then rays associated with the second source, and so on. Define the array manifold matrix $\mathbf{A}_{\theta}$, time manifold matrix $\mathbf{G}_{\tau}$, and path attenuation matrix $\boldsymbol{\Gamma}$ as follows

$$
\begin{aligned}
& \mathbf{A}_{\theta}=\left[\mathbf{a}\left(\theta_{1}\right), \ldots, \mathbf{a}\left(\theta_{r_{1}}\right), \ldots, \mathbf{a}\left(\theta_{r}\right)\right] \in \mathbb{C}^{K \times r} \\
& \boldsymbol{\Gamma}=\operatorname{diag}\left(\beta_{1}, \ldots \beta_{r_{1}}, \ldots, \beta_{r}\right) \in \mathbb{C}^{r \times r} \\
& \mathbf{G}_{\tau}=\left[\mathbf{g}\left(\tau_{1}\right), \ldots, \mathbf{g}\left(\tau_{r_{1}}\right), \ldots, \mathbf{g}\left(\tau_{r}\right)\right] \in \mathbb{C}^{P \times r}
\end{aligned}
$$

where $\mathbf{g}(\tau)=\left[g\left(\frac{1}{P}-\tau\right), \ldots, g\left(1-\frac{1}{P}-\tau\right)\right]^{T} \in \mathbb{C}^{P \times 1} \quad$ is "time manifold" of signals with delay $\tau$. Equation (4) can be simplified to the following formulation [19]

$$
\mathbf{X}=\left[\begin{array}{l}
\mathbf{A}_{\theta} \operatorname{diag}\left(\mathbf{g}\left(\tau_{1}\right)\right) \\
\mathbf{A}_{\theta} \operatorname{diag}\left(\mathbf{g}\left(\tau_{2}\right)\right) \\
\vdots \\
\mathbf{A}_{\theta} \operatorname{diag}\left(\mathbf{g}\left(\tau_{r}\right)\right)
\end{array}\right] \boldsymbol{\Gamma}(\mathbf{S} \mathbf{J})^{T}=\left(\mathbf{G}_{r} \odot \mathbf{A}_{\theta}\right) \mathbf{\Gamma}(\mathbf{S} \mathbf{J})^{T}
$$

where

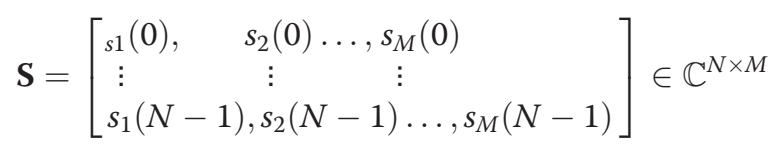

is the transmitted signal matrix and

$$
\mathbf{J}=\left[\begin{array}{ccc}
1_{r_{1}}^{T} & 0, \ldots, & 0 \\
0 & 1_{r_{2}}^{T}, \ldots, & 0 \\
\vdots & \ddots & \\
0 & 0, \ldots, & 1_{r_{M}}^{T}
\end{array}\right]
$$

is a selection matrix that joins multipath associated with a given source. $1_{m}$ denotes an $m \times 1$ vector with elements 1 .

The time delay $\tau$ is usually difficult to estimate from $g(t-\tau)$ directly. Here we map delay $\tau$ into phase shift $\phi$ in the frequency domain by using DFT method. This is a 
classical approach and has been considered in [8]. Assume that $g(t)$ is band limited and the sample rate is at or above the Nyquist rate. Take $P$ points DFT of each antenna output over a single symbol period, and then the follow model is obtained [10]

$$
\overline{\mathbf{X}}=\left(\mathbf{F}_{\phi} \odot \mathbf{A}_{\theta}\right) \boldsymbol{\Gamma}(\mathbf{S J})^{T}
$$

where

$$
\mathbf{F}_{\phi}=\left[\begin{array}{cccc}
1 & 1 & \ldots & 1 \\
\phi_{1} & \phi 2 & \ldots & \phi r \\
\vdots & \vdots & \vdots & \vdots \\
\phi_{1}^{P-1} & \phi_{2}^{P-1} & \ldots & \phi_{r}^{P-1}
\end{array}\right], \phi_{i}=e^{-j 2 \pi \tau_{i} / P}
$$

Note that, by using the DFT method, the delay matrix $\mathbf{G}_{\tau}$ is converted to a Vandermonde matrix.

According to [16], Equation (8) can be viewed as PARALIND model, of which $\mathbf{F}_{\phi}, \mathbf{A}_{\theta}$, and $\mathbf{S}$ are three mode matrices. The selection matrix $\mathbf{J}$ can be viewed as the linear dependence matrix in PARALIND model. Recall that the array response $\mathbf{A}_{\theta}$ and delay matrix $\mathbf{F}_{\phi}$ are both Vandermonde matrices with distinct generator (distinct angle and delay assumption). With the uniqueness property of PARALIND model and the structure property of Vandermonde matrices, $\mathbf{F}_{\phi}$ and $\mathbf{A}_{\theta}$ can be uniquely determined from received data matrix $\overline{\mathbf{X}}$, and DOA and time delay information can be estimated from $\mathbf{F}_{\phi}$ and $\mathbf{A}_{\theta}$.

\section{Uniqueness results}

\section{Partial uniqueness of PARALIND}

The uniqueness of the data model (8) lays the foundation of its applications in parameters estimation. Although PARALIND model can be viewed as an extension of PARAFAC model, uniqueness of PARALIND does not follow directly from the uniqueness property of PARAFAC because of the linear dependence of the loading vectors. PARALIND model usually has only partial uniqueness, which depends on the specifics of the imposed dependency structure along with the adequacy of the factor variation information provided by a given set of data $[16,20]$. Since attenuation matrix $\Gamma$ only leads to the column scaling of $\mathbf{A}_{\theta}$ and $\mathbf{F}_{\phi}$, which does not affect the uniqueness property of the model. Therefore, we simplify $\boldsymbol{\Gamma}$ to be an identity matrix during the following discussion. Then the date model can be written as:

$$
\overline{\mathbf{X}}=\left(\mathbf{F}_{\phi} \odot \mathbf{A}_{\theta}\right)(\mathbf{S J})^{T}
$$

We also use a set of matrices $(\mathbf{X}, \mathbf{A}, \mathbf{B}, \mathbf{C}, \mathbf{H})$ to play the role of $\left(\overline{\mathbf{X}}, \mathbf{F}_{\phi}, \mathbf{A}_{\theta}, \mathbf{S}, \mathbf{J}\right)$, respectively, to simplify the formulations. Equation (10) is converted to the following formulation:

$$
\mathbf{X}=(\mathbf{A} \odot \mathbf{B})(\mathbf{C H})^{T}
$$

PARALIND model has only partial uniqueness, which was first presented in [16]. De Lathauwer has given an essential uniqueness theorem of PARALIND decomposition more quantitatively [21]. The following two concepts are needed.

Definition 1 ( $k$-rank) [22]: Consider a matrix $\mathbf{B} \in \mathbb{C}^{I \times J}$. If $\operatorname{rank}(\mathbf{B})=r$, then $\mathbf{B}$ contains a collection of $r$ linearly independent columns. Moreover, if every $l<J$ columns of $\mathbf{B}$ are linearly independent, but this does not hold for every $l+1$ columns, then $\mathbf{B}$ has $k$-rank $k_{\mathbf{B}}=l$. Note that $k_{\boldsymbol{B}}<\operatorname{rank}(\mathbf{B}), \forall \mathbf{B}$.

Definition 2 ( $k^{\prime}$-rank) [23]: Assume a partitioned matrix $\mathbf{A}=\left[\mathbf{A}_{1}, \ldots, \mathbf{A}_{M}\right]$. The $k^{\prime}$-rank of $\mathbf{A}$, denoted by $\operatorname{rank}_{k^{\prime}}(\mathbf{A})$ or $k_{\mathbf{A}}^{\prime}$, is the maximal number $r$ such that any set of $r$ sub-matrices of $\mathbf{A}$ yields a set of linearly independent columns.

Note that $k^{\prime}$-rank can be viewed as the sub-matrix version of $k$-rank.

Theorem 1 [21]: Let $(\mathbf{A}, \mathbf{B}, \mathbf{C})$ represent a decomposition of $\mathbf{X}$ in (11), where $\mathbf{A}=\left[\mathbf{A}_{1}, \ldots, \mathbf{A}_{M}\right], \mathbf{B}=\left[\mathbf{B}_{1}, \ldots, \mathbf{B}_{M}\right]$ are partitioned matrices with sub-matrices $\mathbf{A}_{m} \in \mathbb{C}^{P \times r_{m}}$, $\mathbf{B}_{m} \in \mathbb{C}^{K \times r_{m}}, m=1, \ldots, M . \mathbf{C}=\left[\mathbf{c}_{1}, \ldots, \mathbf{c}_{M}\right]$ is of $N \times M$ with $\mathbf{c}_{m} \in \mathbb{C}^{N \times 1}, m=1, \ldots, M$. Suppose the condition:

$$
k_{\mathrm{A}}^{\prime}+k_{\mathrm{B}}^{\prime}+k_{\mathrm{C}} \geq 2 M+2
$$

holds and that we have an alternative decomposition of $\mathbf{X}$, represented by $(\hat{\mathbf{A}}, \hat{\mathbf{B}}, \hat{\mathbf{C}})$ with $k_{\hat{\mathbf{A}}}^{\prime}$ and $k_{\hat{\mathbf{B}}}^{\prime}$ maximal under the given dimensionality constraints. Then there holds $\hat{\mathbf{A}}=$ $\mathbf{A} \Pi_{a} \Delta_{a}$ and $\hat{\mathbf{B}}=\mathbf{B} \Pi_{b} \boldsymbol{\Delta}_{b}$, in which $\boldsymbol{\Pi}_{a, b}$ are block permutation matrices and $\boldsymbol{\Delta}_{a, b}$ are square non-singular blockdiagonal matrix, compatible with the block structure of $\mathbf{A}$ and B. It also holds that $\hat{\mathbf{C}}=\mathbf{C} \boldsymbol{\Pi}_{c} \boldsymbol{\Delta}_{c}$, in which $\boldsymbol{\Pi}_{c}$ is a permutation matrix and $\Delta_{c}$ is a diagonal matrix.

Consider Theorem 1 in sub-matrix condition. The ambiguity matrices $\Pi_{a} \Delta_{a}$ and $\Pi_{b} \Delta_{b}$, given in Theorem 1 , can be written as

$$
\begin{aligned}
& \boldsymbol{\Pi}_{a} \Delta_{a}=\operatorname{blockdiag}\left(\mathbf{U}_{1}, \ldots, \mathbf{U}_{M}\right) \\
& \boldsymbol{\Pi}_{b} \boldsymbol{\Delta}_{b}=\operatorname{blockdiag}\left(\mathbf{V}_{1}, \ldots, \mathbf{V}_{M}\right)
\end{aligned}
$$

where $\mathbf{U}_{m} \in \mathbb{C}^{r_{m} \times r_{m}}, \mathbf{V}_{m} \in \mathbb{C}^{r_{m} \times r_{m}}, m=1, \ldots, M$ are $2 M$ non-singular square matrices. Partition $\hat{\mathbf{A}}$ and $\hat{\mathbf{B}}$ to be compatible with the block structure of $\mathbf{A}$ and $\mathbf{B}$, as $\hat{\mathbf{A}}=$ $\left[\hat{\mathbf{A}}_{1}, \ldots, \hat{\mathbf{A}}_{\boldsymbol{M}}\right], \hat{\mathbf{B}}=\left[\hat{\mathbf{B}}_{1}, \ldots, \hat{\mathbf{B}}_{\boldsymbol{M}}\right]$. According to Theorem 1 , it follows 


$$
\begin{aligned}
{\left[\hat{\mathbf{A}}_{1}, \ldots, \hat{\mathbf{A}}_{M}\right] } & =\left[\mathbf{A}_{1}, \ldots, \mathbf{A}_{M}\right] \boldsymbol{\Pi}_{a} \boldsymbol{\Delta}_{a} \\
& =\left[\mathbf{A}_{1}, \ldots, \mathbf{A}_{M}\right] \operatorname{blockdiag}\left(\mathbf{U}_{1}, \ldots, \mathbf{U}_{M}\right) \\
{\left[\hat{\mathbf{B}}_{1}, \ldots, \hat{\mathbf{B}}_{M}\right] } & =\left[\mathbf{B}_{1}, \ldots, \mathbf{B}_{M}\right] \boldsymbol{\Pi}_{b} \boldsymbol{\Delta}_{b} \\
& =\left[\mathbf{B}_{1}, \ldots, \mathbf{B}_{M}\right] \operatorname{blockdiag}\left(\mathbf{V}_{1}, \ldots, \mathbf{V}_{M}\right)
\end{aligned}
$$

and then

$$
\begin{aligned}
\hat{\mathbf{A}}_{m} & =\mathbf{A}_{m} \mathbf{U}_{m}, \hat{\mathbf{B}}_{m}=\mathbf{B}_{m} \mathbf{V}_{m}, m=1, \ldots M \\
\hat{\mathbf{C}} & =\mathbf{C \Pi}_{c} \Delta_{c}
\end{aligned}
$$

\section{Structure uniqueness}

Recalling to data model (10), $\mathbf{F}_{\phi}, \mathbf{A}_{\theta}$, and $\mathbf{S}$ play the roles of $\mathbf{A}, \mathbf{B}$, and $\mathbf{C}$, respectively. According to the partial uniqueness property of PARALIND model, signal matrix $\mathbf{S}$ can be uniquely determined from $\overline{\mathbf{X}}$ up to the permutation and scaling ambiguity. But the array manifold matrix $\mathbf{A}_{\theta}$ and delay matrix $\mathbf{F}_{\phi}$ suffer from rotation ambiguity, which means that $\mathbf{A}_{\theta}$ and $\mathbf{F}_{\phi}$ cannot be uniquely determined without any prior knowledge. Parameters in these two matrices, such as the DOA and the delay information, will not be identifiable directly. But the study [16] has pointed out that PARALIND model can give uniqueness results if some of its mode matrices have theoretically motivated structural constraints. This uniqueness property of PARALIND model is called "structural uniqueness". Since both $\mathbf{A}_{\theta}$ and $\mathbf{F}_{\phi}$ have Vandermonde structure with distinct non-zero generators, the following theorem gives the sufficient condition to restore uniqueness of PARALIND decomposition by capitalizing the property of Vandermonde structure.

Theorem 2 (Structural uniqueness of PARALIND model).

Assume that $\mathbf{A}_{\theta}=\left[\mathbf{a}_{\theta}^{1}, \ldots, \mathbf{a}_{\theta}^{r_{1}}, \ldots, \mathbf{a}_{\theta}^{r}\right] \in \mathbb{C}^{K \times r}, \mathbf{F}_{\phi}=$ $\left[\mathbf{f}_{\phi}^{1}, \ldots, \mathbf{f}_{\phi}^{r_{1}}, \ldots, \mathbf{f}_{\phi}^{r}\right] \in \mathbb{C}^{P \times r}$ are Vandermonde matrices with distinct nonzero generators, and $\mathbf{S}=\left[\mathbf{s}_{1}, \ldots, \mathbf{s}_{M}\right] \in$ $\mathbb{C}^{N \times M} \cdot\left(\mathbf{F}_{\phi}, \mathbf{A}_{\theta}, \mathbf{S}\right)$ represents a decomposition of $\overline{\mathbf{X}}$ in the following formulation:

$$
\overline{\mathbf{X}}=\left(\mathbf{F}_{\phi} \odot \mathbf{A}_{\theta}\right)(\mathbf{S J})^{T}
$$

where $\mathbf{J}$ is the dependence matrix given in (7). $\mathbf{A}_{\theta}$ and $\mathbf{F}_{\phi}$ are partitioned to $M$ sub-matrices: $\mathbf{A}_{\theta}=\left[\mathbf{A}_{\theta}^{1}, \ldots, \mathbf{A}_{\theta}^{M}\right]$, $\mathbf{F}_{\phi}=\left[\mathbf{F}_{\phi}^{1}, \ldots, \mathbf{F}_{\phi}^{M}\right]$, where $\mathbf{A}_{\theta}^{m}=[\underbrace{\underbrace{\sum_{t=1}^{m-1} r_{t}+1}, \ldots, \mathbf{a}_{\theta}^{\sum_{t=1}^{m}} r_{t}} r_{m}]$

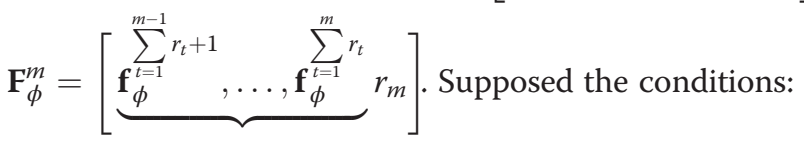

$$
k_{A_{\theta}}^{\prime}+k_{F_{\phi}}^{\prime}+k_{S} \geq 2 M+2
$$

hold and then we have an alternative decomposition of $\overline{\mathbf{X}}$, represented by $\left(\hat{\mathbf{F}}_{\phi}, \hat{\mathbf{A}}_{\theta}, \hat{\mathbf{S}}\right)$ with Vandermonde matrices $\hat{\mathbf{F}}_{\phi}$ and $\hat{\mathbf{A}}_{\theta}$. Partition $\hat{\mathbf{F}}_{\phi}$ and $\hat{\mathbf{A}}_{\theta}$ as:
$\hat{\mathbf{F}}_{\phi}=\left[\hat{\mathbf{F}}_{\phi}^{1}, \ldots, \hat{\mathbf{F}}_{\phi}^{M}\right], \hat{\mathbf{A}}_{\theta}=\left[\hat{\mathbf{A}}_{\theta}^{1}, \ldots, \hat{\mathbf{A}}_{\theta}^{M}\right]$, compatible with the block structure of $\mathbf{F}_{\phi}$ and $\mathbf{A}_{\theta}$ Then the following relationship between $\left(\mathbf{F}_{\phi}, \mathbf{A}_{\theta}, \mathbf{S}\right)$ and $\left(\hat{\mathbf{F}}_{\phi}, \hat{\mathbf{A}}_{\theta}, \hat{\mathbf{S}}\right)$ holds

$$
\begin{aligned}
\hat{\mathbf{S}} & =\mathbf{S} \boldsymbol{\Pi}_{S} \boldsymbol{\Delta}_{S} \hat{\mathbf{A}}_{\theta}^{m}=\mathbf{A}_{\theta}^{m} \boldsymbol{\Pi}_{A_{\theta}}^{m} \Delta_{A_{\theta}}^{m}, \hat{F}_{\phi}^{m} \\
& =\mathbf{F}_{\phi}^{m} \boldsymbol{\Pi}_{F_{\phi}}^{m} \Delta_{F_{\phi}}^{m} \quad m=1, \ldots, M
\end{aligned}
$$

where $\Pi_{A_{\theta}}^{m}, \Pi_{F_{\phi}}^{m}$ and $\Pi_{S}$ are permutation matrices. $\Delta_{A_{\theta}}^{m}, \Delta_{F_{\phi}}^{m}$ and $\Delta_{S}$ are diagonal scaling matrices.

\section{Proof}

Lemma 1 (uniqueness of matrix decomposition with Vandermonde structure) [24]:

Consider a matrix decomposition problem of $\mathbf{X}=\mathbf{A B}^{T}$, in which $\mathbf{A} \in \mathbb{C}^{I \times F}$ is a Vandermonde matrix and $\mathbf{B} \in \mathbb{C}^{J \times F}$ is a non-singular square matrix. Supposed the condition: $I \geq F+1$ is hold and then we have an alternative decomposition of $\mathbf{X}$, represented by $\mathbf{X}=\overline{\mathbf{A}} \overline{\mathbf{B}}^{T}$ with Vandermonde matrix $\overline{\mathbf{A}}$ and non-singular matrix $\overline{\mathbf{B}}$. Then there holds:

$$
\overline{\mathbf{A}}=\mathbf{A} \Pi_{a} \Delta_{a}, \overline{\mathbf{B}}=\mathbf{B} \Pi_{b} \Delta_{b}
$$

where $\Pi_{a}, \Pi_{b}$ are permutation matrices and $\Delta_{a}, \Delta_{b}$ are diagonal scaling matrices with nonzero elements.

According to Theorem 1, if condition $k_{A_{\theta}}^{\prime}+k_{F_{\phi}}^{\prime}+$ $k_{S} \geq 2 M+2$ is satisfied, it holds:

$$
\hat{\mathbf{A}}_{\theta} m=\mathbf{A}_{\theta}^{m} \mathbf{U}_{m}, \hat{\mathbf{F}}_{\phi} m=\mathbf{F}_{\phi}^{m} \mathbf{V}_{m}, m=1, \ldots M
$$

Since $\mathbf{A}_{\theta}^{m}$ and $\mathbf{F}_{\phi}^{m}$ are Vandermonde matrices, $\mathbf{U}_{m}$ and $\mathbf{V}_{m}$ are non-singular square matrices, Lemma 1 provides that $\mathbf{A}_{\theta}^{m}$ can be uniquely decomposed from $\hat{\mathbf{A}}_{\theta}^{m}$ up to the permutation and scaling ambiguity if $K \geq r_{m}+1$. Similarly, $\mathbf{F}_{\phi}^{m}$ can be uniquely decomposed from $\hat{\mathbf{F}}_{\phi}^{m}$ if $P \geq r_{m}+1$. Define $r_{\max }=\max _{m=1, \ldots, M}\left(r_{m}\right)$. When the conditions:

$$
\left\{\begin{array}{l}
K \geq r_{\text {max }}+1 \\
P \geq r_{\text {max }}+1
\end{array}\right.
$$

are satisfied, all of $2 M$ matrices, $\mathbf{A}_{\theta}^{1}, \mathbf{F}_{\phi}^{1}, \ldots, \mathbf{A}_{\theta}^{M}, \mathbf{F}_{\phi}^{M}$, can uniquely determined from $\hat{\mathbf{A}}_{\theta}^{1}, \hat{\mathbf{F}}_{\phi}^{1}, \ldots, \hat{\mathbf{A}}_{\theta}^{M}, \hat{\mathbf{F}}_{\phi}^{M}$.

Now we will show that (13) is a sufficient condition for (15). Recalling the definitions of $k$-rank and $k$ 'rank, the maximal $k^{\prime}$-rank of $\mathbf{A}_{\theta}$ and $\mathbf{F}_{\phi}$ is $M$ and the maximal $k$-rank of $\mathbf{S}$ is also $M$. According to condition (13), it requires that any of $k_{A_{\theta}}^{\prime}, k_{F_{\phi}}^{\prime}$, or $k_{S}$ should be larger than two. By capitalizing on the structure property of Vandermonde matrix, the $k$-rank of $\mathbf{A}_{\theta}$ and $\mathbf{F}_{\phi}$ can be determined easily. Take $\mathbf{A}_{\theta}$ for example, partition $\mathbf{A}_{\theta}$ to $M$ sub-matrices: $\mathbf{A}_{\theta}=\left[\mathbf{A}_{\theta}^{1}, \ldots, \mathbf{A}_{\theta}^{M}\right]$ with $\mathbf{A}_{\theta}^{m} \in \mathbb{C}^{K \times r_{m}}, m=$ $1, \ldots, M$. Reorder $r_{m}$ from large to small and assume 
$r_{i} \geq r_{i+1}, i=1, \ldots, M-1$. The $k^{\prime}$-rank of $\mathbf{A}_{\theta}$ is:

$$
k_{A_{\theta}}^{\prime}=I, \text { where } \sum_{m=1}^{I} r_{m} \leq K \leq \sum_{m=1}^{I+1} r_{m}
$$

With the former assumption, $r_{1}$ is the maximum of $r_{1}, \ldots, r_{M}$, as: $r_{\max }=r_{1}$. If conditions $k_{A_{\theta}}^{\prime} \geq 2$ and $k_{F_{\phi}}^{\prime} \geq 2$ are hold, it follows that:

$$
\left\{\begin{array}{l}
K \geq \sum_{m=1}^{2} r_{m}=r_{1}+r_{2}=r_{\max }+r_{2} \\
P \geq \sum_{m=1}^{2} r_{m}=r_{1}+r_{2}=r_{\max }+r_{2}
\end{array}\right.
$$

Since $r_{2}$ is not less than one (multiple sources assumption), it means that (13) is a sufficient condition for (15). Therefore, (14) is obtained when condition (13) is satisfied.

Notices that in (14), the inherent permutation and scaling ambiguity are still involved, characterized by permutation matrices $\Pi_{A_{\theta}}^{m}, \Pi_{F_{\phi}}^{m}$ and diagonal scaling matrices $\Delta_{A_{\theta}}^{m}, \Delta_{F_{\phi}}^{m}$. Since the elements of the first row of both $\mathbf{A}_{\theta}$ and $\mathbf{F}_{\phi}$ are equal to one, the scaling ambiguity can be easily resolved by normalizing the elements of $\mathbf{A}_{\theta}$ and $\mathbf{F}_{\phi}$ with respect to elements of the first row. The permutation ambiguity will not affect the angle and delay estimation in the proposed algorithm.

\section{Blind JADE algorithm}

In this section, we propose a new blind JADE algorithm based on PARALIND model. The algorithm first uses PARALIND decomposition algorithm to estimate the column space of the sub-matrices of the array manifold matrix $\mathbf{A}_{\theta}$ and delay matrix $\mathbf{F}_{\phi}$. An ESPRIT-like shift-invariance method is then applied to estimate angle and delay parameters.

\section{PARALIND decomposition algorithm}

PARALIND decomposition algorithm is an iterative algorithm based on trilinear alternate least square, which is commonly used to estimate mode matrices of PARAFAC model [25]. The main difference between PARALIND decomposition and PARAFAC decomposition is that the former needs to estimate the dependence matrix $\mathbf{J}$ in (10) if the number of multipath of each source is unknown. The cost function of matrix variables $\mathbf{A}, \mathbf{F}_{\phi}, \mathbf{S}$ and $\mathbf{J}$ is formulated as

$$
f\left(\mathbf{J}, \mathbf{S}, \mathbf{F}_{\phi}, \mathbf{A}_{\theta}\right)=\left\|\overline{\boldsymbol{X}}-\left(\mathbf{F}_{\phi} \odot \mathbf{A}_{\theta}\right)(\mathbf{S J})^{T}\right\|_{F}
$$

Note that the scaling ambiguity matrix $\Gamma$ has been simplified to an identity matrix. This simplification will not affect the decomposition results. By rearranging the received signal matrix $\overline{\mathbf{X}}$, the following two equivalent formulations of PARALIND model are obtained:

$$
\begin{aligned}
& \overline{\boldsymbol{Y}}=\left((\mathbf{S} \mathbf{J}) \odot \mathbf{F}_{\phi}\right) \mathbf{A}_{\theta}^{T} \\
& \overline{\boldsymbol{Z}}=\left(\mathbf{A}_{\theta} \odot(\mathbf{S J})\right) \mathbf{F}_{\phi}^{T}
\end{aligned}
$$

According to (10), rearrange $\overline{\mathbf{X}}$ to a vector:

$$
\begin{aligned}
\overline{\boldsymbol{x}} & =\operatorname{vec}(\overline{\boldsymbol{X}})=\operatorname{vec}\left(\left(\mathbf{F}_{\phi} \odot \mathbf{A}_{\theta}\right) \mathbf{J}^{T} \mathbf{S}^{T}\right) \\
& =\left(\mathbf{S} \otimes \mathbf{F}_{\phi} \odot \mathbf{A}_{\theta}\right) \operatorname{vec}\left(\mathbf{J}^{T}\right)
\end{aligned}
$$

Here, we have used the general relation: $\operatorname{vec}(\mathbf{A B C})=$ $\left(\mathbf{C}^{T} \otimes \mathbf{A}\right) \operatorname{vec}(\mathbf{B})$. Then $\mathbf{J}$ can be obtained in least square sense:

$$
\mathbf{J}=\arg \min _{J}\left\|\overline{\boldsymbol{x}}-\left(\mathbf{S} \otimes \mathbf{F}_{\phi} \odot \mathbf{A}_{\theta}\right) \operatorname{vec}\left(\mathbf{J}^{T}\right)\right\|_{F}
$$

\begin{tabular}{|c|c|}
\hline Step 1: & Initialization \\
\hline & Initialize matrices $\mathbf{J}^{(0)}, \mathbf{A}_{\theta}^{(0)}, \mathbf{F}_{\varphi}^{(0)}$ and $\mathbf{S}^{(0)}, k=0$ \\
\hline Step 2: & $k=k+1$ \\
\hline Step 3: & $\begin{array}{l}\text { Update } \boldsymbol{J} \text { based on (21), given } \mathbf{S}^{(k)}, \mathbf{F}_{\varphi}^{(k)} \text { and } \mathbf{A}_{\theta}^{(k)} \text { : } \\
\mathbf{j}^{(k+1)}=\left(\mathbf{S}^{(k)} \otimes \mathbf{F}_{\varphi}^{(k)} \odot \mathbf{A}_{\theta}^{(k)}\right)^{\dagger} \overline{\mathbf{x}} \\
\mathbf{J}^{(k+1)}=\text { unvec }\left(\mathbf{j}^{(k+1)}\right)\end{array}$ \\
\hline Step 4: & $\begin{array}{l}\text { Update } \mathbf{S} \text { based on (22), given } \mathbf{F}_{\varphi}^{(k)}, \mathbf{A}_{\theta}^{(k)} \text { and } \mathbf{J}^{(k+1)} \text { : } \\
\mathbf{S}^{(k+1)}=\left(\left(\mathbf{F}_{\varphi}^{(k)} \odot \mathbf{A}_{\theta}^{(k)}\right)\left(\mathbf{J}^{(k+1)}\right)^{T}\right)^{\dagger} \overline{\mathbf{X}}\end{array}$ \\
\hline Step 5: & $\begin{array}{l}\text { Update } \mathbf{F}_{\varphi} \text { based on (23), given } \mathbf{A}_{\theta}^{(k)}, \mathbf{S}^{(k+1)} \text { and } \mathbf{J}^{(k+1)} \text { : } \\
\mathbf{F}_{\varphi}^{(k+1)}=\left(\mathbf{A}_{\theta}^{(k)} \odot\left(\mathbf{S}^{(k+1)} \mathbf{J}^{(k+1)}\right)\right)^{\dagger} \overline{\mathbf{Z}}\end{array}$ \\
\hline Step 6: & $\begin{array}{l}\text { Update } \mathbf{A}_{\theta} \text { based on (24), given } \mathbf{S}^{(k+1)}, \mathbf{J}^{(k+1)} \text { and } \mathbf{F}_{\varphi}^{(k+1)} \text { : } \\
\mathbf{A}_{\theta}^{(k+1)}=\left(\left(\mathbf{S}^{(k+1)} \mathbf{J}^{(k+1)}\right) \odot \mathbf{F}_{\varphi}^{(k+1)}\right)^{\dagger} \overline{\mathbf{Y}}\end{array}$ \\
\hline Step 7: & $\begin{array}{l}\text { Calculate the fitting residual value: } \\
\boldsymbol{\varepsilon}^{(k+1)}=\|\left(\mathbf{F}_{\phi}^{(k+1)} \odot \mathbf{A}_{\theta}^{\left(k+\|_{F}^{1}\right)}\right)\left(\mathbf{S}^{(k+1)} \mathbf{J}^{(k+1)}\right)^{T}- \\
\left(\mathbf{F}_{\varphi}^{(k)} \odot \mathbf{A}_{\theta}^{(k)}\right)\left(\mathbf{S}^{(k)} \mathbf{J}^{(k)}\right)^{T} \\
\text { repeat step 2-7 until }\left|\boldsymbol{\varepsilon}^{(k+1)}-\boldsymbol{\varepsilon}^{(k)}\right|<1 e^{-8 .}\end{array}$ \\
\hline
\end{tabular}

Similarly, $\mathbf{S}, \mathbf{F}_{\phi}$ and $\mathbf{A}_{\theta}$ can be obtained based on (10), (19), and (20)

$$
\begin{aligned}
& \mathbf{S}=\arg \min _{S}\left\|\overline{\boldsymbol{X}}-\left(\mathbf{F}_{\phi} \odot \mathbf{A}_{\theta}\right)(\mathbf{S J})^{T}\right\|_{F} \\
& \mathbf{F}_{\phi}=\arg \min _{\mathbf{F}_{\phi}}\left\|\overline{\boldsymbol{Z}}-\left(\mathbf{A}_{\theta} \odot(\mathbf{S J})\right) \mathbf{F}_{\phi}^{T}\right\|_{F}
\end{aligned}
$$

\section{Table 1 PARALIND decomposition algorithm}




$$
\mathbf{A}_{\theta}=\arg \min _{\mathbf{A}_{\theta}}\left\|\overline{\boldsymbol{Y}}-\left((\mathbf{S J}) \odot \mathbf{F}_{\phi}\right) \mathbf{A}_{\theta}^{T}\right\|_{F}
$$

$\mathbf{J}, \mathbf{S}, \mathbf{F}_{\phi}$ and $\mathbf{A}_{\theta}$ are updated alternatively in least square sense until results converge. Assume that ()$^{(k)}$ stands for the value of the $k$ th iteration. Let $\mathbf{j}=\operatorname{vec}(\mathbf{J})$. PARALIND decomposition algorithm is presented in Table 1.

In the noisy condition, the estimation of the dependent matrix, denoted as $\hat{\mathbf{J}}$, is not a strict selection matrix with elements of 1 and 0 . There are two methods to reconstruct $\hat{\mathbf{J}}$ to be a standard selection matrix. A simple method to resolve this problem is to define a threshold and use hard decision to reconstruct $\hat{\mathbf{J}}$. If the element of $\hat{\mathbf{J}}$ is more than the threshold, project it to 1 , while if the element is less than the threshold, project it to 0 . This method is simple and has good performance in high SNR condition. But, in low SNR condition, the variance of estimation is large and the value of threshold is hard to be determined. Note that the main work of $\mathbf{J}$ is to provide the structure of the multipath of each user. Therefore, we only need to recovery this structure from $\hat{\mathbf{J}}$ (not to determine its the elements). Many clustering methods can be used to recovery the structure. Simulation results show that this method can work well in both low and high SNR conditions.

\section{ESPRIT-like shift-invariance technique}

According to the partial uniqueness theorem of PARALIND decomposition, when PARALIND decomposition algorithm is finished, data matrix $\mathbf{S}$ can be obtained from received signal matrix $\overline{\mathbf{X}}$. Array manifold matrix $\mathbf{A}_{\theta}$ and delay matrix $\mathbf{F}_{\phi}$ still suffer from rotation ambiguity. Assume that $\hat{\mathbf{A}}_{\theta}, \hat{\mathbf{F}}_{\phi}$ and $\hat{\mathbf{S}}$ are the estimation of $\mathbf{A}_{\theta}, \mathbf{F}_{\phi}$ and S. Partition $\hat{\mathbf{F}}_{\phi}$ and $\hat{\mathbf{A}}_{\theta}$ as $\hat{\mathbf{F}}_{\phi}=\left[\hat{\mathbf{F}}_{\phi}^{1}, \ldots, \hat{\mathbf{F}}_{\phi}^{M}\right], \hat{\mathbf{A}}_{\theta}=$ $\left[\hat{\mathbf{A}}_{\theta}^{1}, \ldots, \hat{\mathbf{A}}_{\theta}^{M}\right]$, compatible with the block structure of $\mathbf{F}_{\phi}$ and $\mathbf{A}_{\theta}$. Rewrite (14)

$$
\hat{\mathbf{S}}=\mathbf{S} \Pi \Delta \hat{\mathbf{A}}_{\theta} m=\mathbf{A}_{\theta}^{m} \mathbf{U}_{m}, \hat{\mathbf{F}}_{\phi}^{m}=\mathbf{F}_{\phi}^{m} \mathbf{V}_{m}, m=1, \ldots M
$$

Capitalizing on the Vandermonde structure of $\mathbf{A}_{\theta}^{m}$ and $\mathbf{F}_{\phi}^{m}, \theta$, and $\tau$ can be uniquely determined from $\hat{\mathbf{A}}_{\theta}^{m}$ and $\hat{\mathbf{F}}_{\phi}^{m}$ when Theorem 2 is satisfied. Here, we use an ESPRIT-like shift-invariance technique and eigenvalue decomposition method to estimate angle and delay of the multiray from each source. Take the angle estimation procedure for example. We collect the first $K-1$ and the last $K-1$ rows of $\hat{\mathbf{A}}_{\theta}^{m}$ to construct two sub-matrix $\hat{\mathbf{A}}_{\theta, 1}^{m}$ and $\hat{\mathbf{A}}_{\theta, 2}^{m}$. Define $\mathbf{A}_{\theta, 1}^{m}$ as the sub-matrix including first $K-1$ rows of $\mathbf{A}_{\theta}^{m}$. According to the shift-invariance property of date model: $\hat{\mathbf{A}}_{\theta}^{m}=\mathbf{A}_{\theta}^{m} \mathbf{U}_{m}$, we have the following results:

$$
\hat{\mathbf{A}}_{\theta, 1}^{m}=\mathbf{A}_{\theta, 1}^{m} \mathbf{U}_{m} \hat{\mathbf{A}}_{\theta, 2}^{m}=\mathbf{A}_{\theta, 1}^{m} \operatorname{diag}\left(\mathbf{D}_{m}\right) \mathbf{U}_{m}
$$

where

$$
\mathbf{D}_{m}=\left[e^{-j \frac{2 \pi d}{\lambda} \sin \left(\theta_{m, 1}\right)}, \ldots, e^{-\frac{j 2 \pi d}{\lambda} \sin \left(\theta_{m, r_{m}}\right)}\right]^{T}
$$

is a vector that includes angle information of all $r_{m}$ paths of source $m$. Angles of paths from source $m$ can be estimated from as follows

$$
\begin{aligned}
\hat{\boldsymbol{\theta}}_{m} & =\left[\hat{\theta}_{m, 1}, \ldots, \hat{\theta}_{m, r_{m}}\right]^{T} \\
& =\arcsin \left(\frac{\operatorname{imag}\left(-\operatorname{In}\left(\hat{\mathbf{D}}_{m}\right)\right) \lambda}{2 \pi d}\right)
\end{aligned}
$$

$\hat{\mathbf{D}}_{m}$ is the estimation of $\mathbf{D}_{m}$. Now the problem is simplified to one that how to estimate $\hat{\mathbf{D}}_{m}$ from $\hat{\mathbf{A}}_{\theta, 1}^{m}$ and $\hat{\mathbf{A}}_{\theta, 2}^{m}$. Let

$$
\left(\hat{\mathbf{A}}_{\theta, 1}^{m}\right)^{\dagger} \hat{\mathbf{A}}_{\theta, 2}^{m}=\mathbf{U}_{m}^{-1} \operatorname{diag}\left(\mathbf{D}_{m}\right) \mathbf{U}_{m}
$$

According to the definition of eigenvalue decomposition, $\mathbf{D}_{m}$ is the eigenvalue of $\left(\hat{\mathbf{A}}_{\theta, 1}^{m}\right)^{\dagger} \hat{\mathbf{A}}_{\theta, 2}^{m}$, formulated as

$$
\hat{\mathbf{D}}_{m}=\operatorname{eigs}\left(\left(\hat{\mathbf{A}}_{\theta, 1}^{m}\right)^{\dagger} \hat{\mathbf{A}}_{\theta, 2}^{m}\right)
$$

where eigs () is the eigenvalue decomposition operator. The delay estimation procedure is similar to the angle estimation. The angle and delay estimation procedure are presented in Tables 2 and 3.

Note that the angles and delays of rays of each source can be estimated separately based on

$$
\hat{\mathbf{A}}_{\theta}^{m}=\mathbf{A}_{\theta}^{m} \mathbf{U}_{m}, \hat{\mathbf{F}}_{\phi}^{m}=\mathbf{F}_{\phi}^{m} \mathbf{V}_{m}, m=1, \ldots M
$$

\section{Table 2 Angle estimation procedure}

$$
\begin{aligned}
& \text { Loop: } \\
& m=1 \text { to } M \\
& \text { Construct sub-matrices } \hat{\mathbf{A}}_{\theta, 1}^{m} \text { and } \hat{\mathbf{A}}_{\theta, 2}^{m} \\
& \hat{\mathbf{A}}_{\theta, 1}^{m}=\hat{\mathbf{A}}_{\theta}^{m}(1: K-1,:) \hat{\mathbf{A}}_{\theta, 2}^{m}=\hat{\mathbf{A}}_{\theta}^{m}(2: K,:) \\
& \text { Calculate eigenvalue of }\left(\hat{\mathbf{A}}_{\theta, 1}^{m}\right)^{\dagger} \hat{\mathbf{A}}_{\theta, 2}^{m} \hat{\mathbf{D}}_{m} \\
& =\operatorname{eigs}\left(\left(\hat{\mathbf{A}}_{\theta, 1}^{m}\right)^{\dagger} \hat{\mathbf{A}}_{\theta, 2}^{m}\right) \\
& \text { Calculate the estimation of angles } \\
& \hat{\theta}_{m}=\left[\hat{\theta}_{m, 1}, \ldots, \hat{\theta}_{m, r_{m}}\right]^{T} \\
& =\arcsin \left(\frac{\operatorname{imag}\left(-\ln \left(\hat{\mathbf{D}}_{m}\right)\right) \lambda}{2 \pi d}\right)
\end{aligned}
$$


Table 3 Delay estimation procedure

$$
\begin{aligned}
& \text { Loop: } m=1 \text { to } M \\
& \begin{array}{l}
\mathrm{m}=1 \text { to } M \\
\text { Construct sub-matrices } \hat{\mathbf{F}}_{\phi, 1}^{m} \text { and } \hat{\mathbf{F}}_{\theta, 2}^{m} \hat{\mathbf{F}}_{\phi, 1}^{m}=\hat{\mathbf{F}}_{\phi}^{m}(1: K-1,:) \\
\hat{\mathbf{F}}_{\phi, 2}^{m}=\hat{\mathbf{F}}_{\phi}^{m}(2: K,:)
\end{array} \\
& \text { Calculate eigenvalue of }\left(\hat{\mathbf{F}}_{\phi, 1}^{m}\right)^{\dagger} \hat{\mathbf{F}}_{\phi, 2}^{m} \hat{\mathbf{D}}_{m}=\operatorname{eigs}\left(\left(\hat{\mathbf{F}}_{\phi, 1}^{m}\right)^{\dagger} \hat{\mathbf{F}}_{\phi, 2}^{m}\right) \\
& \text { Calculate the estimation of delays } \hat{\boldsymbol{\tau}}_{m}=\left[\hat{\boldsymbol{\tau}}_{m, 1}, \ldots, \hat{\boldsymbol{\tau}}_{m, r_{m}}\right]^{\top} \\
& =P \frac{-\operatorname{imag}\left(\ln \left(\hat{\mathbf{D}}_{m}\right)\right)}{2 \pi}
\end{aligned}
$$

end loop

Table 4 Angles and delays of multipath rays of sources

\begin{tabular}{lll}
\hline & $\begin{array}{l}\text { Number of } \\
\text { multipath }\end{array}$ & $\begin{array}{l}\text { Angle and delay of each } \\
\text { path (angle, delay) }\end{array}$ \\
\hline Source 1 & Three paths & $\left(5^{\circ}, 0.5 \mathrm{Tp}\right),\left(15^{\circ}, 1.5 \mathrm{Tp}\right),\left(25^{\circ}, 2.5 \mathrm{Tp}\right)$ \\
Source 2 & Two paths & $\left(35^{\circ}, 3.5 \mathrm{Tp}\right),\left(45^{\circ}, 4.5 \mathrm{Tp}\right)$ \\
Source 3 & Two paths & $\left(55^{\circ}, 5.5 \mathrm{Tp}\right),\left(65^{\circ}, 6.5 \mathrm{Tp}\right)$ \\
\hline
\end{tabular}

which implies that the PARALIND decomposition method can convert the multiuser-multipath parameters estimation problem to a simple singleuser-multipath problem. Therefore, the proposed PARALIND-based estimation algorithm can distinguish the estimated parameters of each source automatically, which is usually not obtained in conventional JADE algorithm.

\section{Simulations}

In this section, we evaluate the performance of proposed algorithm, referred to as PARALIND-based angle and delay estimation algorithm (PARALIND-JADE). Complex additive Gaussian white noise $\mathbf{E}_{X}$ is added

$$
\overline{\boldsymbol{X}}=\left(\mathbf{F}_{\phi} \odot \mathbf{A}_{\theta}\right) \mathbf{\Gamma}(\mathbf{J} \mathbf{S})^{T}+\mathbf{E}_{X}
$$

SNR is defined in terms of the noisy data model (25):

$$
\boldsymbol{S N R}=10 \log _{10}|| \overline{\boldsymbol{X}}||_{F}^{2} /|| \mathbf{E}_{X} \|_{F}^{2} \boldsymbol{d B}
$$

$M=3$ sources are used in the following simulation. There are totally seven rays in the receiving end. The parameters of the multiple rays of each source are listed in Table 4. where $\mathrm{Tp}$ is the sampling time interval. Oversampling factor $P$ is equal to 10 in the following simulations. Some other simulation parameters, such as the number of snapshots $N$, the number of antennae $K$, and SNR, are varied in each simulation. Traditional blind JADE algorithm proposed in [10] is also simulated for comparison, which is refered here as Expanded JADE-ESPRIT (E-JADE-ESPRIT). E-JADE-ESPRIT is a type of subspace-based algorithm which uses temporal/ spatial smoothing and joint diagonalization to achieve angle and delay estimation.

\section{Simulation 1}

The performances of angle and delay estimation of PARALIND-JADE and E-JADE-ESPRIT algorithms are evaluated in this simulation. The number of receiving antennae $K=10$, oversampling factor $P=10$ and the number of snapshots $N=100$. Five hundred independent Monte Carlo
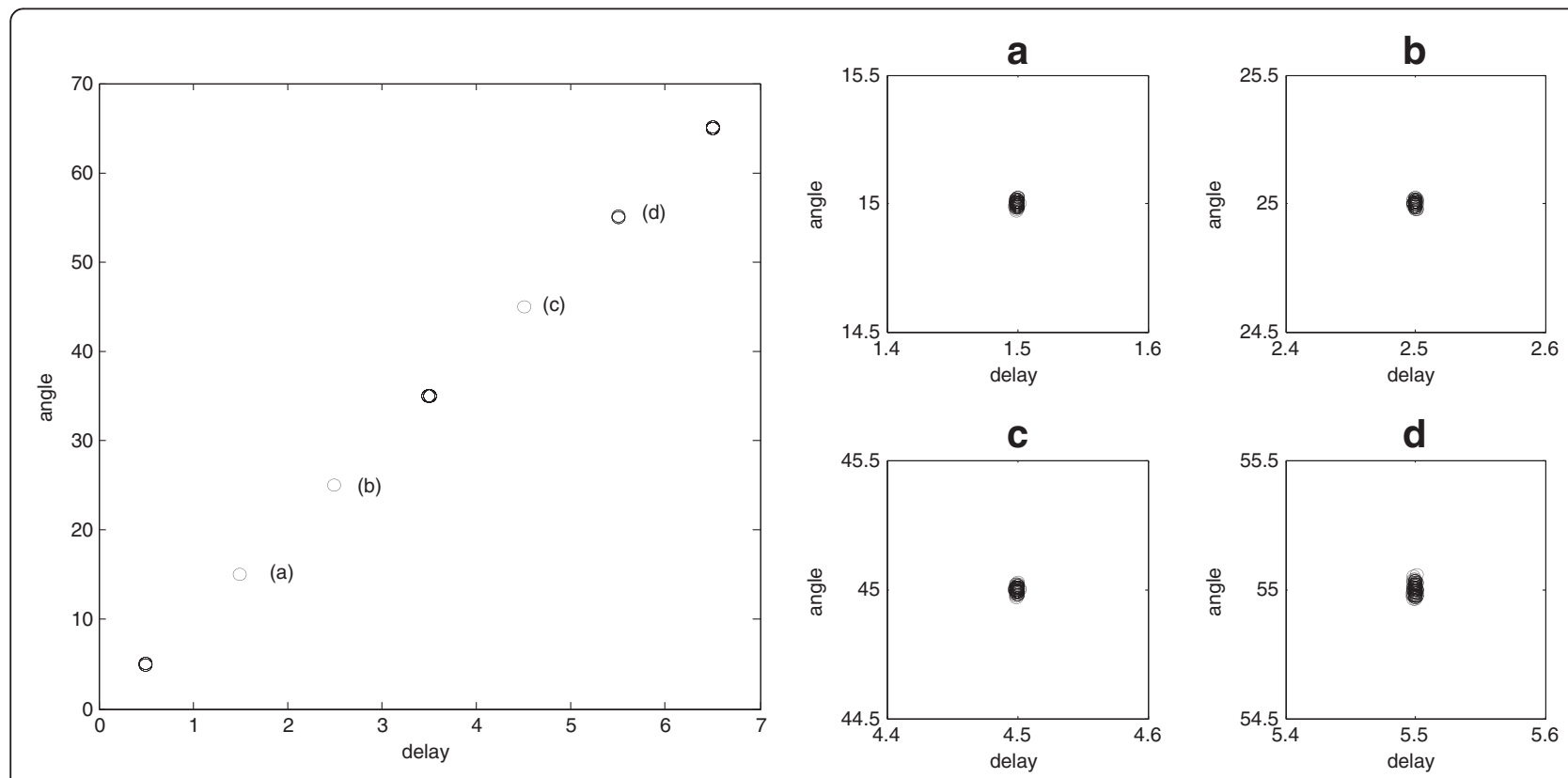

Figure 2 Delay-angle scatter of PARALIND-JADE algorithm, SNR $=30 \mathrm{~dB}$. 
Lu et al. EURASIP Journal on Advances in Signal Processing 2012, 2012:130

Page 9 of 13 http://asp.eurasipjournals.com/content/2012/1/130

a

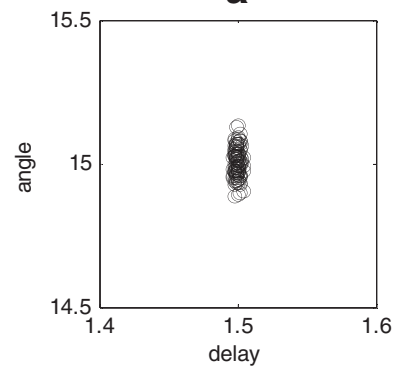

C

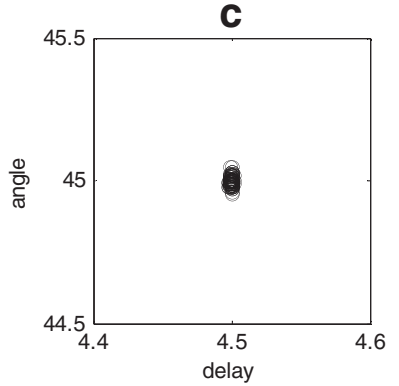

b

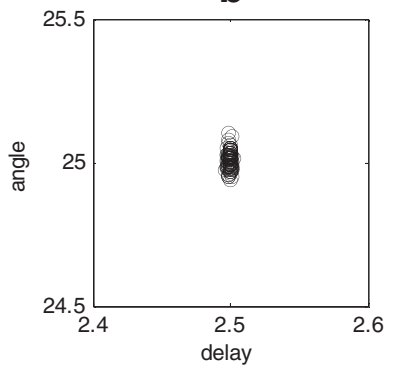

d

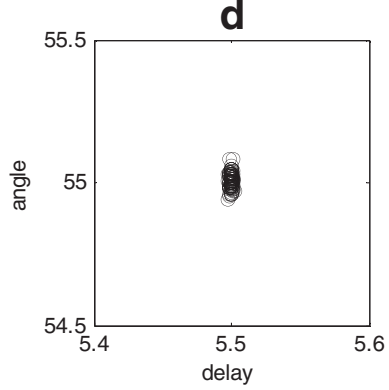

Figure 3 Delay-angle scatter of E-JADE-ESPRIT algorithm, SNR $=30 \mathrm{~dB}$.

a

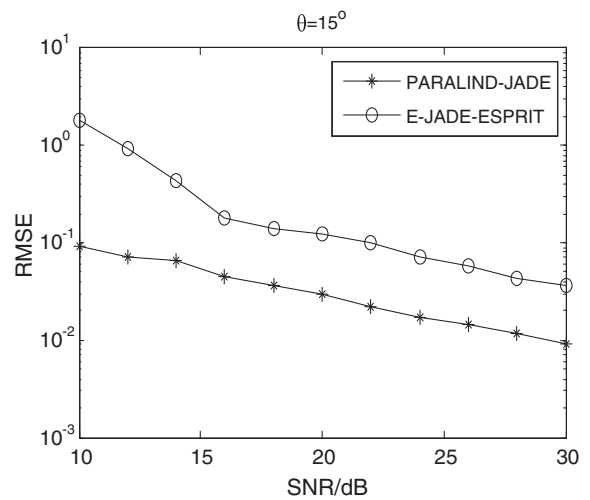

C

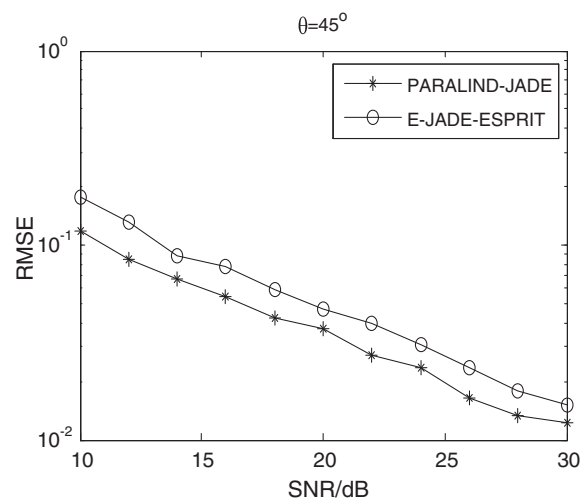

b

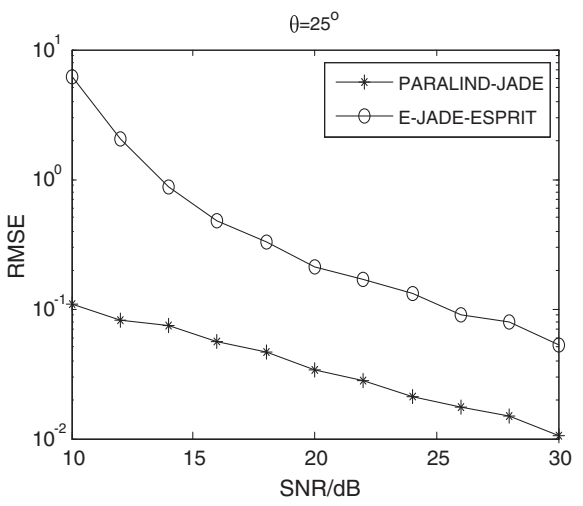

d

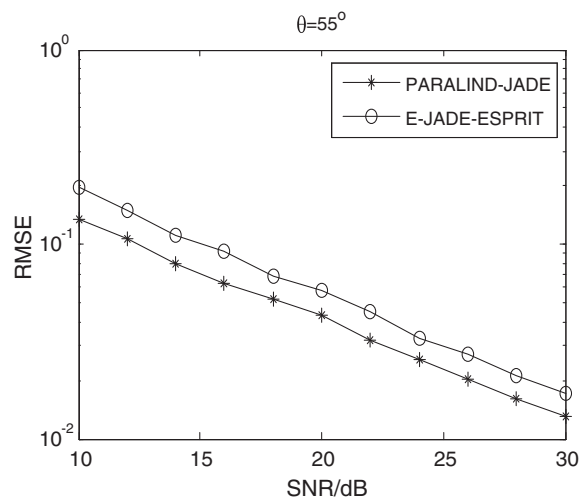

Figure 4 RMSE curve of angle estimation versus SNR. 
runs are used to evaluate the performance. Figures 2 and 3 present the delay-angle scatter diagram of PARALINDJADE and E-JADE-ESPRIT algorithms $(\mathrm{SNR}=30 \mathrm{~dB})$. The $X$ coordinate stands for delay and the $Y$ coordinate stands for angle. The left sub-figure of each depicts the estimated angle-delay diagram of all seven paths, and the right four sub-figures, titled as (a), (b), (c), (d), are the zoom-in diagrams of angle and delay estimation of four paths with parameters $\left(15^{\circ}, 1.5 \mathrm{Tp}\right),\left(25^{\circ}, 2.5 \mathrm{Tp}\right),\left(45^{\circ}, 4.5 \mathrm{Tp}\right)$ and $\left(55^{\circ}\right.$, $5.5 \mathrm{Tp})$, respectively. It is clear that the degree of divergence of the diagrams in Figure 2 is smaller than that in Figure 3. Therefore, the proposed algorithm performs better than EJADE-ESPRIT in parameters estimation.

\section{Simulation 2}

This experiment evaluates the root mean squared error (RMSE) of the two algorithms in angle and delay estimation. Here, we order all multipath rays listed in Table 4 as 1-7. The RMSE values of the estimated angle and delay of the $j$ th ray are calculated as follows:

$$
\boldsymbol{R} \boldsymbol{M S} \boldsymbol{E}_{\text {angle }}^{(j)}=\sqrt{\frac{1}{Q} \sum_{i}^{Q}\left(\hat{\theta}_{i}^{(j)}-\theta^{(j)}\right)^{2} \|_{F}^{2}, j=1, \ldots, 7}
$$

$$
\boldsymbol{R M S E}_{\text {delay }}^{(j)}=\sqrt{\frac{1}{Q} \sum_{i}^{Q}\left(\hat{\tau}_{i}^{(j)}-\tau^{(j)}\right)^{2} \|_{F}^{2}, j=1, \ldots, 7}
$$

where $\hat{\theta}_{i}(j)$ and $\hat{\tau}_{i}(j)$ are the estimation of angle $\theta^{(j)}$ and delay $\tau^{(j)}$ of $j$ th ray in $i$ th simulation. $Q$ is the number of Monte Carlo simulations. There are 10 antennae in the receiving antennae array, and the number of snapshots $N=100$. SNR varies from 10 to $30 \mathrm{~dB}$. Five hundred independent Monte Carlo runs are simulated. Figures 4 and 5 present the RMSE curve of angle and delay estimations versus SNR. Four sub-figures of each figure, titled as (a), (b), (c), (d), depict performance of four rays with parameters $\left(15^{\circ}, 1.5 \mathrm{Tp}\right),\left(25^{\circ}\right.$, $2.5 \mathrm{Tp}),\left(45^{\circ}, 4.5 \mathrm{Tp}\right)$, and $\left(55^{\circ}, 5.5 \mathrm{Tp}\right)$. The RMSE values of parameter estimation of PARALIND-JADE are less than that of E-JADE-ESPRIT. It implies that the proposed algorithm has better accuracy in parameter estimation.

\section{Simulation 3}

The RMSE performances of angle and delay estimations with different number of snapshots are evaluated in this simulation. The number of snapshots $N$ varies a

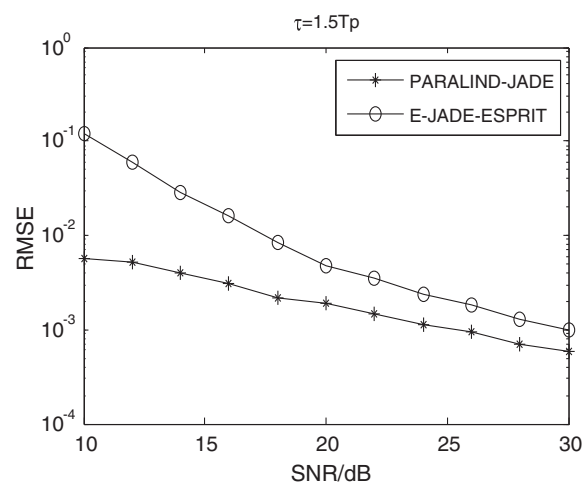

C

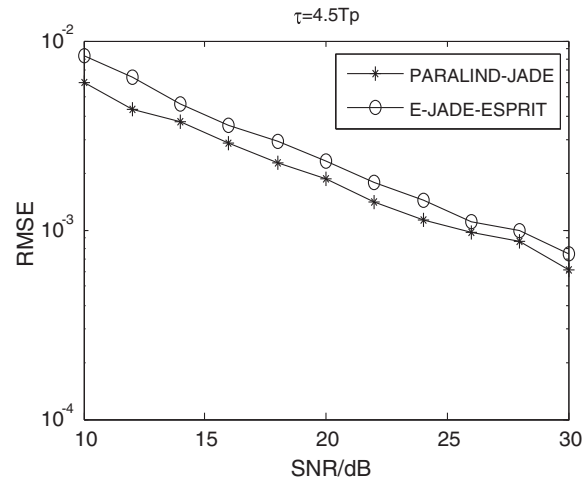

b

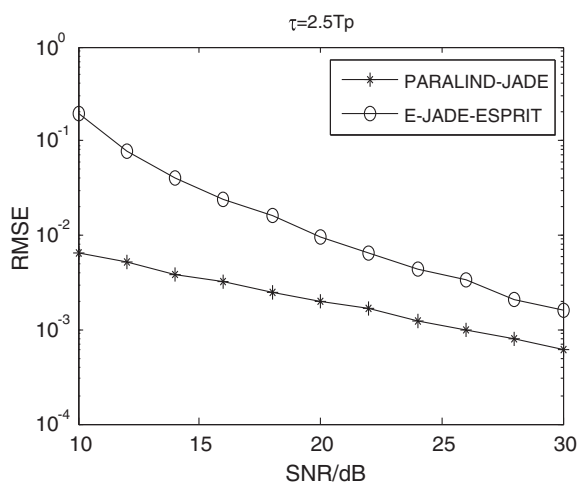

d

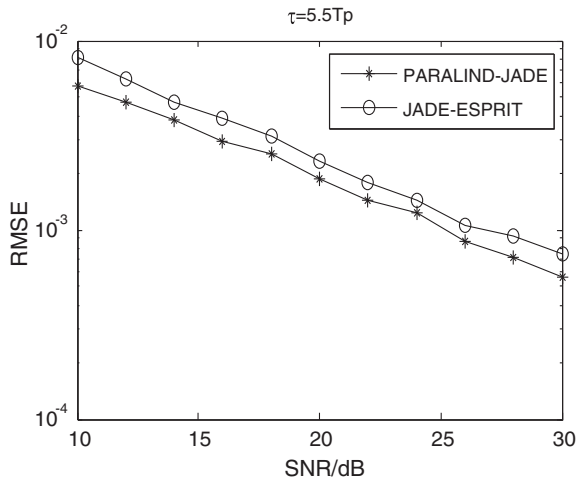

Figure 5 RMSE curve of delay estimation versus SNR. 

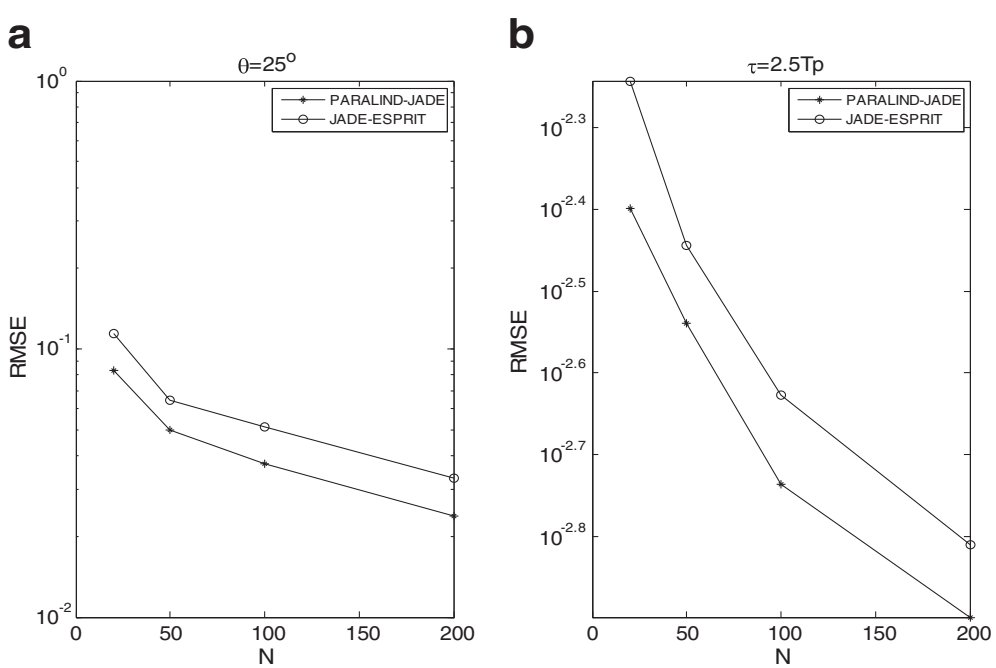

Figure 6 RMSE curve of angle and delay estimation versus the number of snapshots.

from 20 to 200. The number of antennae $K=10$ and $\mathrm{SNR}=30 \mathrm{~dB}$. Take the second ray with parameters $\left(25^{\circ}, 2.5 \mathrm{Tp}\right)$ for example (the performance of other rays is similar), Figure 6 depicts the simulation results. It is shown that the RMSE performance of PARALINDJADE algorithm is improved and always better than that of E-JADE-ESPRIT algorithm as the number of snapshots increases.

\section{Simulation 4}

In the last simulation, we evaluate the RMSE performances of angle and delay estimation with different
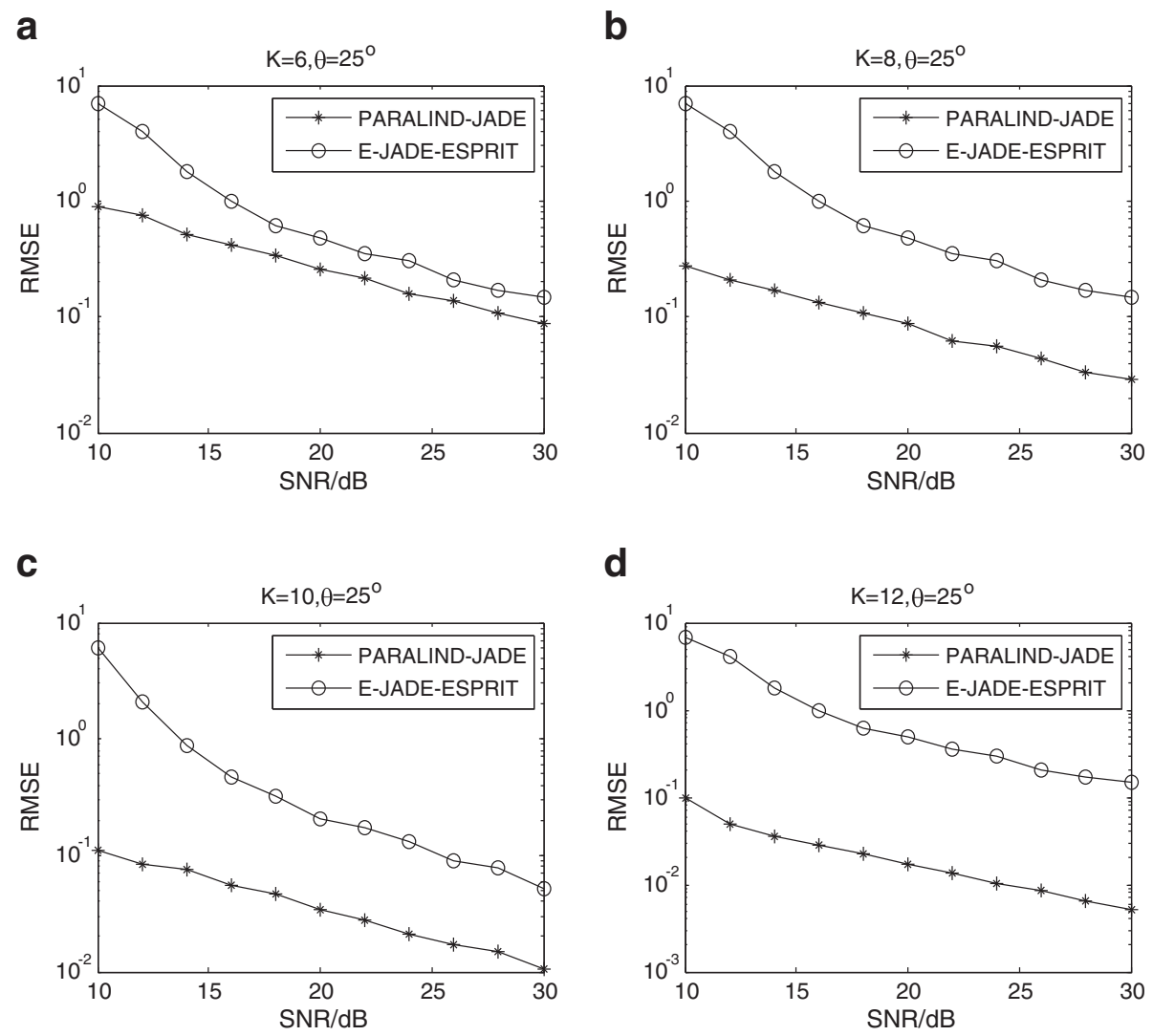

Figure 7 RMSE curve of angle estimation versus SNR $(K=6,8,10,12)$. 
a

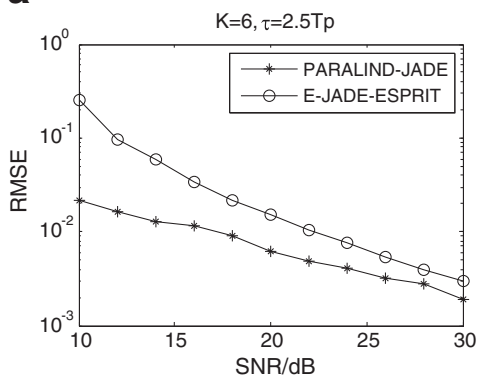

C

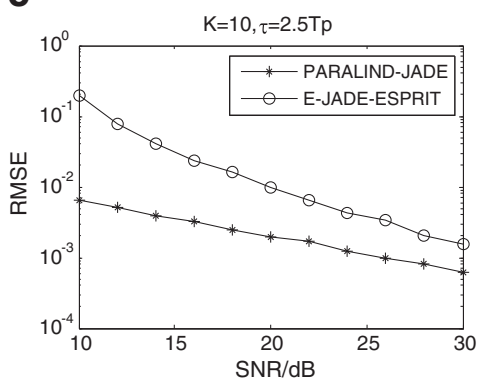

b

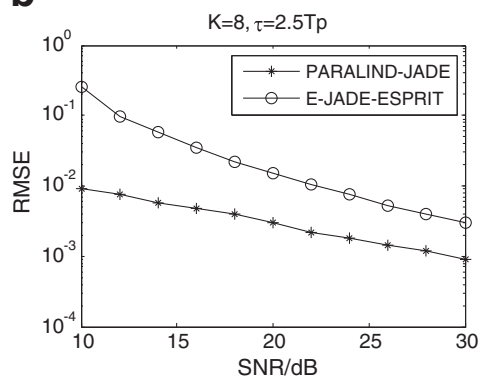

d

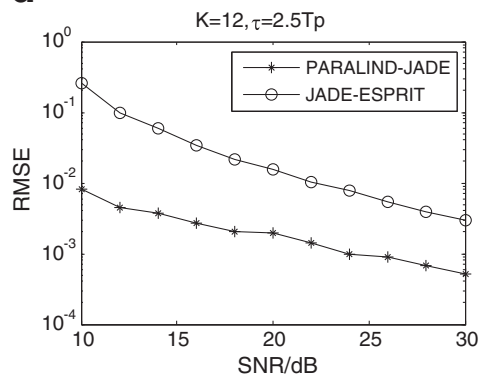

Figure 8 RMSE curve of delay estimation versus SNR $(K=6,8,10,12)$.

number of receiving antennae. The number of antennae varies from 6 to 12. The number of snapshots $N=100$ and SNR varies from 10 to $30 \mathrm{~dB}$. Figure 7 depicts the RMSE curve of angle estimation versus SNR of the ray with parameters $\left(25^{\circ}, 2.5 \mathrm{Tp}\right)$ in different number of receiving antennae scenario $(K=6,8,10,12)$, and Figure 8 gives the related RMSE curve of delay estimation. Notice that the proposed algorithm still works well when the number of multiple rays is larger than the number of receiving antennae $(K=6)$.

\section{Conclusions}

This article links the blind JADE problem to PARALIND analysis. A new PARALIND-based JADE algorithm (PARALIND-JADE) is proposed based on the PARALIND decomposition algorithm and an ESPRIT-like shiftinvariance technique. The structural uniqueness result of PARALIND model with Vandermonde structure is also presented. Simulation shows that the proposed algorithm performs better than traditional JADE algorithm. It can automatically distinguish the estimated parameters of each source and still work well when the number of rays is larger than the number of receiving antennae.

\section{Endnote}

${ }^{a}$ Although PARAFAC analysis is still available to multipath CDMA signals, it should follow the assumption that the multipath delay should much smaller than the symbol period [11].

\section{Competing interests}

The authors declare that they have no competing interests.

\section{Acknowledgments}

This study was supported by the China NSF Grants (61101104, 61071090), National Science and Technology Major Project (2011ZX03005-004-03), Jiangsu "973" Project (BK2011027), Jiangsu Planned Projects for Postdoctoral Research Funds (1201035C) Nanjing University of Posts \&

Telecommunications Project (NY211010). The authors wish to thank the anonymous reviewers for their valuable suggestions on improving this article.

\section{Author details}

${ }^{1}$ Jiangsu Key Laboratory of Wireless Communication, College of Telecommunications and Information Engineering, Nanjing University of Posts \& Telecommunications, Nanjing, China. ${ }^{2}$ Department of Information Technology, University of Turku, Turku, Finland.

Received: 22 December 2011 Accepted: 17 May 2012

Published: 3 July 2012

\section{References}

1. JH Winters, Smart antenna for wireless systems. IEEE Personal Commun. 5(1), 23-27 (1998)

2. B Li, D Xie, S Cheng, J Chen, P Zhang, W Zhu, B Li, Recent advances on TDSCDMA in China. IEEE Commun. Mag. 41(6), 116-119 (2005)

3. K Fatih, C Hasari, G Sinan, AQ Khalid, A Huseyin, HV Poor, Time-delay estimation in dispersed spectrum cognitive radio systems. EURASIP J. Adv. Signal Process (2010). doi:10.1155/2010/675959

4. M Tabs, T Fusco, A Petrella, Joint symbol timing and CFO estimation for OFDM/OQAM systems in multipath channels. EURASIP J. Adv. Signal Process (2010). doi:10.1155/2010/897607

5. M Feder, E Weinstein, Parameter estimation of superimposed signals using the EM algorithm. IEEE Trans. Acoust. Speech, Signal. Process 36(4), 477-489 (1998)

6. B Fleury, M Tschudin, R Heddergott, D Dahlhaus, PK Ingeman, Channel parameter estimation in mobile radio environments using the SAGE algorithm. IEEE J. Sel. Areas Commun. 17(3), 434-450 (1999) 
7. M Zoltowski, M Haardt, C Mathews, Closed-form 2-d angle estimation with rectangular arrays in element space or beamspace via unitary ESPRIT. IEEE Trans. Signal Process. 44(2), 316-328 (1996)

8. AJV der Veen, MC Vanderveen, A Paulraj, Joint angle and delay estimation using shift-invariance techniques. IEEE Trans. Signal Process. 46(2), 405-418 (1998)

9. MC Vanderveen, AJV der Veen, Estimation of multipath parameters in wireless communications. IEEE Trans. Signal Process. 46(3), 682-690 (1998)

10. AJV der Veen, Algebraic methods for deterministic blind beamforming. Proc. IEEE 86(10), 1987-2008 (1998)

11. ND Sidiropoulos, G.B. Giannakis, R. Bro, Blind PARAFAC receivers for DSCDMA systems. IEEE Trans. Signal Process. 48(3), 810-822 (2000)

12. ALF De Almeida, G Favier, JCM Mota, Constrained tensor modeling approach to blind multiple-antenna CDMA schemes. IEEE Trans. Signal Process. 56(6), 2417-2428 (2008)

13. ND Sidiropoulos, R Bro, GB Giannakis, Parallel factor analysis in sensor array processing. IEEE Trans. Signal Process. 48(8), 2377-2388 (2000)

14. XF Zhang, B Feng, DZ Xu, Blind joint symbol detection and DOA estimation for OFDM system with antenna array. Wirel. Personal Commun. 46(3), 371-383 (2008)

15. CER Fernandes, G Favier, JCM Mota, Blind channel identification algorithm based on the Parafac decomposition of cumulant tensors: the single and multiuser cases. Signal Process 88, 1382-1401 (2008)

16. R Bro, RA Harshman, ND Sidiropoulos, ME Lundy, Modeling multi-way data with linearly dependent loadings. J. Chemometr. 23(7-8), 324-340 (2009)

17. ND Sidiropoulos, GZ Dimie, Blind multiuser detection in W-CDMA systems with large delay spread. IEEE Signal Process. Lett. 8(3), 87-89 (2001)

18. XF Zhang, X Gao, Z Wang, Blind PARALIND multiuser detection for smart antenna CDMA system over multipath fading channel. Prog. Electromag. Res. 89, 23-38 (2009). doi:23

19. ND Sidiropoulos, $X$ Liu, Identifiability results for blind beamforming in incoherent multipath with small delay spread. IEEE Trans. Signal Process. 49(1), 228-238 (2001)

20. T Khayamian, GH Tan, A Sirhan, YF Siew, SM Sajjadi, Comparison of three multi-way models for resolving and quantifying bifenthrin and tetramthrin using gas chromatography-mass spectrometry. Chemometr. Intell. Lab. Syst. 96, 149-158 (2009)

21. LD Lathauwer, Decompositions of a higher-order tensor in block termsPart 2: definitions and uniqueness. SIAM J. Matrix Anal. Appl. 30(3), 1033-1066 (2008)

22. JB Kruskal, Three-way arrays: rank and uniqueness of trilinear decompositions, with application to arithmetic complexity and statistics. Linear Algebra Appl. 18, 95-138 (1977)

23. LD Lathauwer, Decompositions of a higher-order tensor in block termsPart 1: lemmas for partitioned matrices. SIAM J. Matrix Anal. Appl. 30(3), 1022-1032 (2008)

24. X Liu, ZZ Xu, L Lei, Identifiability results for array signal parameters based on matrix decomposition. J. Appl. Sci. (China) 28(1), 49-55 (2010)

25. R Bro, PARAFAC: tutorial and applications. Chemometr. Intell. Lab. Syst. 38, 149-171 (1997)

\section{Submit your manuscript to a SpringerOpen ${ }^{\circ}$ journal and benefit from:}

- Convenient online submission

- Rigorous peer review

- Immediate publication on acceptance

- Open access: articles freely available online

High visibility within the field

- Retaining the copyright to your article

Submit your next manuscript at $\gg$ springeropen.com 